\title{
Modeling longitudinal data on health aging and life span
}

\author{
A.I. Yashin*, K.G. Arbeev, I. Akushevich, A. Kulminski, S.V. Ukraintseva, E. Stallard, \\ K.C. Land
}

Center for Population Health and Aging, Duke University, Durham, NC 27708, USA

Available online 26 May 2012

Communicated by L. Perlovsky

\begin{abstract}
We address comments from the three discussants of our paper, paying particular attention to the properties of our model likely to be of interest in new applications to complex dynamic systems.

(c) 2012 Elsevier B.V. All rights reserved.
\end{abstract}

Keywords: Stochastic process model of aging and mortality; Longitudinal data analysis; Allostatic load; Stress resistance; Physiological norms

We are grateful to the commentators for the attention and effort they made to read and discuss various aspects of the target paper [1]. Their comments will play an important role in the future development of the methodology of analyzing longitudinal data on aging, health, and longevity.

Tolley [2] drew attention to the importance of introducing and investigating the feedback mechanisms regulating age trajectories of physiological variables. This type of mechanism is crucial for biological systems. It also plays a key role in dynamic automatic control technical systems which tend to deteriorate over time and, eventually, fail. The quadratic hazard/stochastic process model can be adjusted for investigating factors and mechanisms of deterioration as well. This is because its dynamic part describes the evolution of variables playing key roles in the functioning of specific systems under study. The importance of including a feedback mechanism in the model of aging is that it allows us to address aging-related changes in mechanisms responsible for the decline in adaptive capacity. The importance of this property for living organisms was emphasized by Rose [3] among others.

It is also important to note that the dynamics of average trajectories of physiological variables in the quadratic hazard model are regulated by two feedback mechanisms. One describes the homeostatic regulation of individual trajectories which tends to keep the physiological index close to the value of the variable describing the effect of allostatic adaptation. Another mechanism regulates the average age trajectory by keeping it close to the trajectory of normal values of the corresponding physiological index. This mechanism describes the effects of mortality selection on the average age trajectory of each physiological variable [4].

DOI of original article: http://dx.doi.org/10.1016/j.plrev.2012.05.002.

DOI of comments: http://dx.doi.org/10.1016/j.plrev.2012.05.008, http://dx.doi.org/10.1016/j.plrev.2012.05.006, http://dx.doi.org/10.1016/j. plrev.2012.05.007.

* Corresponding author.

E-mail address: aiy@duke.edu (A.I. Yashin). 
The fact that the occurrence of many chronic diseases tends to increase with age indicates the possible contribution of aging in the development and manifestation of such diseases. Detailed analyses of age patterns of incidence rates of major human diseases reveal that after an accelerated increase at the beginning these rates tend to decelerate, level off, and then decline at the old and oldest old ages. Mitnitski and Rockwood [5] proposed that the quadratic hazard model can be used not only for studying aging to better understand mechanisms of mortality and survival, but also for understanding the regularities of disease development and the proper organization of health care. We agree. The methods of quantifying aging-related changes used in our approach can be used for analyzing disease development. For this purpose instead of dealing with mortality, one has to deal with the disease rates in the model, using data on age at disease onset instead of lifespan data. The results of such analyses will make important contributions to the development of effective preventive and treatment measures. Recently, this idea was further developed in an extended stochastic process model constructed for the joint analyses of data on individual health histories and age trajectories of physiological indices [6,7]. We also applied the quadratic hazard model for evaluating the effects of health care on population health [8].

Mitnitski and Rockwood [5] also called attention to the importance of incorporating information accumulated in the fields of epidemiology and biology of aging into the structure of the stochastic process model. This is an important capability of our model which distinguishes it from many other models and approaches used in analyzing longitudinal data with informative censoring or in joint analyses of longitudinal and time-to-event data (see references in [1]). Our approach has been successful in addressing questions about biological mechanisms regulating agingrelated changes in physiological biomarkers [4,9]. The approach has been recently extended to involve processes developing at molecular biological levels in individual organisms which may lead to cancer [10] or other diseases. The approach may be used for linking other biological processes developing at the individual level in aging organisms with population manifestations of these changes.

The stochastic process model discussed in [1] has been developed to show that using biological knowledge in statistical analyses of longitudinal data is efficient for aging studies. The fact that we reviewed only models developed and used by our group does not mean that other approaches are not suitable for studying the process of aging and developing aging related diseases. Many models and methods developed by other research groups can be extended and used in analyses of longitudinal data to address research questions of high scientific and practical importance. For example, the "degradation" model developed by Nikulin and Bagdonavicius [11-14] links an individual's chances of survival with the process of deterioration developing in a complex system which could be a living organism or technical device. The statistical properties of many other methods of analyses of longitudinal data have been more fully investigated than those of the model described in [1]. Since the goal of our paper was to inform researchers about a new promising direction of research and invite them to think about developing a tool capable of dealing with an abundance of available information about aging, diseases, and longevity, we were not able to review other methods which also may potentially benefit the research field.

The focus of our on-going analyses is to identify factors and evaluate mechanisms regulating aging and its consequences for health and survival of living organisms. We consider the mathematical model of aging we described as a tool which has to be convenient to use and capable of addressing more sophisticated research questions than alternative statistical models which do not incorporate biological information about aging into the model structure. The model itself can be further modified and adjusted for application to different data.

A property of our model of longitudinal data which is useful in applications to analyses of health or survival outcomes is its ability to deal with random times at which the age trajectory stops or disappears (in the case of mortality), wherein the stopping-time distribution depends on age dependent variables (e.g., the case of "informative censoring"). In probability and stochastic process theory, this was referred to as "killing times" [15] or in martingale literature as "stopping times" [16]. As Tolley [2] mentioned, Woodbury used the term "manholes", meaning that the age trajectories of variables describing each individual's state can be drawn only until his or her time of death. The model admits extensions to situations with sequences of health-related events, where the age trajectories of physiological variables experience jumps at the time of disease onset. Using such models, individual health histories can be jointly analyzed with age trajectories of biomarkers measured in longitudinal studies of aging, health, and longevity $[6,7,17]$.

Finkelstein [18] in his comments nicely described other possibilities for taking stochastically changing covariates into account. He also explained an advantage to our approach of dealing with the quadratic hazard instead of "first passage time" used in several other applications [19]. Although this advantage is technical, it adds a lot of flexibility 
to our approach. Partly it is because the dimension of the biological processes involved in aging is large, so the boundary in physiological space distinguishing between life and death is not observed when one deals with a low dimensional approximation of the reality. The end of life resulting from reaching an unobserved boundary in high dimensional space will be represented by the "manholes" in the space of lower dimension. Another useful application of the stochastic process model with a quadratic hazard is to study dependence among competing risks by allowing the same components of variables describing the physiological state to influence the onset of different diseases [20].

Many systems around us undergo an "aging" or deterioration process over time which affects the quality of their functioning. Studying factors and mechanisms of degradation of such systems as well as factors affecting their chances of failure is important for maintaining proper functioning of these systems. We hope that the discussion of our paper will generate new insights and ideas about how to properly address issues of aging-related degradation and failure in complex dynamic systems.

\section{References}

[1] Yashin AI, Arbeev KG, Akushevich I, Kulminski A, Ukraintseva SV, Stallard E, et al. The quadratic hazard model for analyzing longitudinal data on aging, health, and the life span. Physics of Life Reviews 2012;9(2):177-88 [in this issue].

[2] Tolley HD. Using the random walk model for understanding aging. Physics of Life Reviews 2012;9(2):191-2 [in this issue].

[3] Rose MR. The long tomorrow: how advances in evolutionary biology can help us postpone aging. New York, USA: Oxford University Press; 2005.

[4] Yashin AI, Arbeev KG, Akushevich I, Ukraintseva SV, Kulminski A, Arbeeva LS, et al. Exceptional survivors have lower age trajectories of blood glucose: lessons from longitudinal data. Biogerontology 2010;11:257-65.

[5] Mitnitski A, Rockwood K. Advancing our understanding of aging using mathematical modeling of longitudinal data. Physics of Life Reviews 2012;9(2):193-4 [in this issue].

[6] Yashin AI, Akushevich I, Arbeev KG, Kulminski A, Ukraintseva SV. Joint analysis of health histories, physiological states, and survival. Mathematical Population Studies 2011;18:207-33.

[7] Yashin AI, Akushevich I, Arbeev KG, Kulminski A, Ukraintseva SV. New approach for analyzing longitudinal data on health, physiological state, and survival collected using different observational plans. In: JSM proceedings, section on government statistics. Alexandria, VA: American Statistical Association; 2011. p. 5336-50.

[8] Yashin AI, Arbeev KG, Kulminski A, Akushevich I, Akushevich L, Ukraintseva SV. What age trajectories of cumulative deficits and medical costs tell us about individual aging and mortality risk: findings from the NLTCS-Medicare data. Mech Ageing Dev 2008;129:191-200.

[9] Yashin AI, Ukraintseva SV, Arbeev KG, Akushevich I, Arbeeva LS, Kulminski AM. Maintaining physiological state for exceptional survival: what is the normal level of blood glucose and does it change with age? Mech Ageing Dev 2009;130:611-8.

[10] Akushevich I, Veremeyeva G, Kravchenko J, Ukraintseva S, Arbeev K, Akleyev AV, et al. New stochastic carcinogenesis model with covariates: an approach involving intracellular barrier mechanisms. Math Biosci 2012;236:16-30.

[11] Bagdonavicius V, Nikulin M. Life models A. Boca Raton: Chapman and Hall/CRC; 2002.

[12] Bagdonavicius V, Nikulin M. Semiparametric analysis of degradation and failure time data with covariates. In: Nikulin MS, Balakrishnan N, Mesbah M, Limnios N, editors. Parametric and semiparametric models with applications to reliability, survival analysis, and quality of life. Boston: Birkhauser; 2004. p. 41-65.

[13] Bagdonavicius V, Bikelis A, Kazakevicius V, Nikulin M. Non-parametric estimation in degradation-renewal-failure models. In: Nikulin M, Commenges D, Huber C, editors. Probability, statistics and modelling in public health. New York: Springer; 2006. p. 23-36.

[14] Bagdonavicius V, Nikulin M. Statistical models to analyze failure, wear, fatigue, and degradation data with explanatory variables. Communications in Statistics - Theory and Methods 2009;38:3031-47.

[15] Dynkin EB. Theory of Markov processes. New Jersey, USA: Prentice \& Hall Inc.; 1961.

[16] Liptser RS, Shiryayev AN. Theory of martingales. Dordrecht, The Netherlands: Kluwer Academic Publishers; 1989.

[17] Yashin AI, Arbeev KG, Ukraintseva SV, Akushevich I, Kulminski A. Patterns of aging related changes on the way to 100: an approach to studying aging, mortality, and longevity from longitudinal data. In: 2011 living to 100 monograph Society of Actuaries Monograph M-LI11-1. Schaumburg, IL: Society of Actuaries; 2011.

[18] Finkelstein M. The Gompertz law of mortality and longitudinal data. Physics of Life Reviews 2012;9(2):189-90 [in this issue].

[19] Aalen OO, Borgan O, Gjessing HK. Survival and event history analysis: a process point of view. New York, USA: Springer; 2008.

[20] Yashin AI, Manton KG, Stallard E. Dependent competing risks: a stochastic process model. J Math Biol 1986;24:119-40. 